\title{
Eficacia de una intervención en alfabetización basada en la evidencia para niños hispanoparlantes con riesgo lector de niveles socioeconómicos vulnerables
}

\section{Alejandra Balbi ${ }^{1}$, Alexa von Hagen ${ }^{2}$, Juan E. Jimenez ${ }^{3}$, Ariel Cuadro ${ }^{1}$}

${ }^{1}$ Facultad de Psicología, Universidad Católica del Uruguay

${ }^{2}$ National Institute of Education,

Nanyang Technological University, Singapore

${ }^{3}$ Facultad de Psicología, Universidad de La Laguna

\section{Uruguay}




\section{Resumen}

Introducción. Las investigaciones apoyan la eficacia de las intervenciones en alfabetización basadas en la evidencia para estudiantes angloparlantes con riesgo lector, pero no queda claro si resultados similares se pueden conseguir con alumnos hispanoparlantes.

Método. Investigamos el impacto de una intervención en alfabetización basada en la evidencia con una duración de 15 horas, en niños con riesgo lector de $1^{\circ}$ y $2^{\circ}$ año de escuela de niveles socioeconómicos (NSE) vulnerables en Uruguay, con español como lengua materna. Los estudiantes con riesgo lector fueron asignados aleatoriamente a una intervención $(n=68)$ suplementaria a la instrucción usual de aula o a un grupo de control $(n=57)$ y se compararon con otro grupo de desarrollo lector típico $(n=69)$ en medidas pre-post-test en conciencia fonológica, escritura, fluidez y comprensión

Resultados. Aunque todos los participantes mostraron mejoras significativas en relación a su trayectoria pre-post-test, los niños con riesgo lector que participaron de la intervención experimental solamente mostraron mayores niveles de crecimiento que el grupo control en la habilidad para identificar rimas y parcialmente en comprensión lectora.

Discusión y conclusiones. Los hallazgos de este estudio reflejan la necesidad de tomar en cuenta los contrastes entre los sistemas de escritura del español y el inglés, así como el perfil cognitivo de niños de NSE vulnerables en Latinoamérica a la hora de aplicar prácticas basadas en la evidencia al diseño de programas de intervención para niños con riesgo lector de niveles socioeconómicos vulnerables en países de habla hispana.

Palabras Clave: alfabetización temprana, intervención, conciencia fonológica, prácticasbasadas-en-la-evidencia, nivel socioeconómico bajo. 


\begin{abstract}
Introduction. Past research supports the efficacy of evidence-based literacy interventions for English-speaking struggling readers, but it remains unknown if similar results can be achieved in Spanish.

Methods. We assessed the impact of a 15-hour long evidence-based literacy intervention for Spanish speaking struggling readers attending grade 1 and 2 in primary schools of vulnerable socio-economic status (SES) in Uruguay. Struggling readers were randomly assigned to a supplementary intervention $(n=68)$ in addition to business-as-usual classroom instruction or control group $(n=57)$ and compared to an additional group of typically developing readers $(n$ = 69) on pre-post measures of phonological awareness, spelling, reading fluency and comprehension.
\end{abstract}

Results. Although all participants showed significant improvements with respect to on pre-post trajectories, struggling readers in the intervention group only achieved higher growth levels as compared to the control group on rhyme identification and partially for comprehension skills.

Discussion and conclusions. Findings from this study underline the need to consider contrasts between the writing systems of Spanish and English, as well as cognitive profiles of children from vulnerable SES in Latin-America when applying evidence-based practices to design intervention programs for struggling readers from low SES in Spanish speaking countries.

Keywords: early literacy, intervention, phonological awareness, evidence-based practices, low socio-economic status 


\section{Introducción}

Educadores e investigadores coinciden en que el campo de la alfabetización debe fusionar saberes teóricos e intervención basada en la evidencia. Desafortunadamente, la mayoría de estas contribuciones provienen de participantes angloparlantes, por lo que no queda claro si estas intervenciones basada en la evidencia tendrán un impacto similar en otras lenguas (Escamilla, Loera, Ruiz y Rodríguez, 1998; Mathes, Pollard-Durodola, Cárdenas-Hagan, Linan-Thompson y Vaughn, 2007; Share, 2008).

En ortografías más transparentes, como el español, la mayoría de las palabras pueden ser leídas a través de asignaciones biunívocas entre grafemas y fonemas. Por el contrario, en ortografías más profundas, las reglas de correspondencia grafema-fonema no siempre son fácilmente predecibles y en muchos casos admiten más de una transcripción dependiendo de la palabra (Kohnen, Colenbrander, Krajenbrink y Nickels, 2015). Varios estudios translingüísticos han abordado estas diferencias (Caravolas et al., 2012; Moll et al., 2014) y llegan a la conclusión de que es probable que el impacto de las intervenciones de alfabetización en poblaciones angloparlantes sea diferente en lenguas distintas del inglés. Más aún, advierten que esto tenga importantes implicaciones prácticas para el diseño e implementación de las intervenciones de alfabetización en idiomas distintos del inglés (Escamilla et al., 1998; Mathes et al., 2007).

Un ejemplo de las diferencias respecto a la eficacia de las intervenciones en alfabetización fue proporcionado por Ehri, Nunes, Willows, Yaghoub-Zadeh y Shanahan (2001) en un meta-análisis centrado en el impacto de la instrucción en conciencia fonológica. Los autores encontraron valores de impacto significativamente más altos en inglés que en ortografías más superficiales en varias medidas de alfabetización: la conciencia fonológica (inglés: $d=0.99$; otras lenguas: $d=0.65$ ), lectura (inglés: $d=.63$; otras lenguas: $d=.36$ ) y la ortografía (inglés: $d=0,95$; otras lenguas: $d=0,51$ ). Dentro del grupo de lenguas distintas al inglés, Ehri et al. (2001) incluyeron noruego, finlandés, sueco, danés, español, hebreo, holandés y alemán, que difieren en gran medida entre sí con respecto a la consistencia de sus correspondencias grafema-fonema. Por tanto, es posible que un estudio de la eficacia de una intervención en alfabetización centrado solamente en el español (Defior, Jiménez-Fernández, Calet y Serrano, 2015), podría revelar resultados aún más distantes que la evidencia indicada por Ehri et al. (2001). 
Furnes y Samuelson (2010) informaron que la conciencia fonológica sólo contribuyó significativamente en la explicación de las habilidades de lectura en el primer año de escuela primaria en estudiantes suecos y noruegos, mientras que en inglés siguió siendo un predictor poderoso hasta el segundo año de la escuela primaria. Por otra parte, en griego, Tafa y Manolitsis (2008) no encontraron ninguna contribución significativa de la conciencia fonológica que distinga entre lectores riesgo y promedio. A su vez, diferentes ortografías también podrían presentar variaciones en la incidencia, ya no de la conciencia fonológica como un todo, sino en las diferentes sub-habilidades de conciencia fonológica. Mientras que las habilidades de rima desempeñan un papel importante en las ortografías más opacas, unidades fonológicas más pequeñas como las sílabas y fonemas, parecen ser más importantes en español (Goodwin, Agosto y Calderón, 2015).

En español, la evidencia actual de la eficacia de las intervenciones en alfabetización muestra resultados diversos. Estudios realizados en los EE.UU. con hablantes nativos de español apoyan un impacto positivo de las intervenciones de alfabetización adaptadas de programas de inglés existentes como es el caso de "Reading Recovery (Escamilla et al., 1998). En forma similar Mathes et al. (2007) completaron estudios paralelos en español e inglés e informan que la versión española de la intervención, "Lectura Proactiva" tuvo tanto éxito como la versión en inglés.

Los escasos reportes de estudios realizados en países de habla hispana en general brindan información sobre la eficacia de intervenciones en alfabetización centradas en mejorar la calidad de instrucción en el aula usual por medio de programas de formación docente. Así, por ejemplo, el programa "Un buen comienzo" evidenció una mejora significativa de la calidad de la alfabetización, pero sin diferencias significativas entre el grupo de tratamiento y el grupo control. Yoshikawa et al. (2015) explicaron este resultado señalando que la observación de la instrucción en el aula mostró que las actividades de lectura sólo aumentaron 15 minutos en el grupo de tratamiento, en comparación con el grupo control. Los autores especulan que esta baja dosis de las actividades de alfabetización podría explicar la falta de un efecto de tratamiento específico.

Por otra parte, Pallante y Kim (2013) encontraron un impacto significativo en alumnos chilenos del jardín de infantes, pero no en estudiantes de primer año de escuela primaria que participaron de una intervención de alfabetización de un año llamada Collaborative Language 
and Literacy Instruction Project (CLLIP) enfocada a mejorar la instrucción en el aula regular. Además, los estudiantes de jardín de infantes de bajo nivel socioeconómico (NSE) mostraron una curva de crecimiento más lenta en comparación con sus compañeros de clase de NSE alto. Strasser, Rolla y Romero-Contreras (2016) enfatizan el NSE como un factor que podría explicar las diferencias en el impacto observado entre las intervenciones de alfabetización en inglés y español. Se especula que los niños categorizados en bajo NSE participantes en estudios latinoamericanos podrían presentar déficits lingüísticos más profundos que los niños incluidos en esta misma categoría en estudios similares en países de habla inglesa.

El objetivo del presente estudio fue examinar la eficacia de una intervención de alfabetización suplementaria a la instrucción usual de aula con niños de habla hispana de NSE bajo en Uruguay. Estábamos interesados en investigar en qué medida una intervención de alfabetización diseñada sobre principios basados en la evidencia derivados de investigaciones con poblaciones de habla inglesa también sería adecuada para mejorar las habilidades de alfabetización en niños de NSE bajo en un país de habla hispana. Nos propusimos como objetivos: (1) evaluar el progreso de los participantes que recibieron nuestra intervención en alfabetización con medidas pre-post-test en conciencia fonológica, escritura, fluidez lectora y comprensión lectora; y también (2) investigar en qué medida este progreso podía atribuirse específicamente a nuestro programa de intervención mostrando mejores resultados que la 'enseñanza usual' del aula.

\section{Método}

\section{Participantes}

Estudiantes $(n=483)$ de primer y segundo año de seis escuelas primarias privadas de Montevideo participaron en este estudio; fueron clasificadas en NSE bajo porque: (a) estaban situados en los barrios de NSE bajo según la clasificación del Instituto Nacional de Estadísticas (INE, 2012b) el importe máximo de pago mensual esperado por los padres no superó el valor de \$ 20 (dólares estadounidenses - UCU, 2012). Eran todos hablantes nativos de español sin exposición a otros idiomas, con la excepción de la entrada ocasional en inglés a través de programas de televisión o música. Fueron evaluados en una tarea de lectura de letras y palabras. Los que obtuvieron un resultado menor al percentil 25 fueron asignados al azar al grupo experimental $(n=68)$ que participó de nuestro programa de intervención o al grupo control ( $n$ 
= 57). Los niños con resultados entre los percentiles 40-60 fueron asignados a un tercer grupo $(n=69)$.

\section{Instrumentos}

Identificación de Letras y Palabras de la Batería III Woodcock-Muñoz (MuñozSandoval, Woodcock, McGrew y Mather, 2005a). La consistencia interna es $r=0.95$ basada en una muestra de personas de habla hispana en los EE.UU., España y diferentes países de América Latina (Schrank, McGrew, Ruef, Alvarado, Muñoz-Sandoval y Woodcock, 2005). Desafortunadamente, no existen normas uruguayas disponibles para esta prueba.

Matrices (Raven, 1993). Esta medida se empleó para evaluar habilidades de razonamiento no verbal. El instrumento cuenta con una consistencia interna de $\alpha=.84$ en base a una muestra de niños de Chile (Jara Quezada y Troncoso San Martín, 2014). Desafortunadamente, no existen normas uruguayas disponibles para esta prueba.

Comprensión oral. Esta medida se evaluó a través de la subprueba de comprensión oral de la Batería III Woodcock-Muñoz Test (Muñoz-Sandoval et al., 2005a). La consistencia interna se informa como $r=.93$ (Schrank et al., 2005).

Conciencia fonológica. La subprueba de Discernimiento de Sonidos de la Batería III, Woodcock-Muñoz (Muñoz-Sandoval et al., 2005a) se utilizó para medir esta capacidad. Esta subprueba discrimina diferentes sub-habilidades: rima, cancelación, sustitución e inversión de fonemas. La consistencia interna es $r=.94$ (Schrank et al., 2005).

Escritura de palabras. Se utilizó la subprueba de Ortografía de la Batería III WoodcockMuñoz Test (Muñoz-Sandoval et al., 2005a) para evaluar esta capacidad. La consistencia interna es $r=.93$ (Schrank et al., 2005).

Fluidez lectora. Para medir fluidez lectora se administró la Prueba de Eficacia de lectura - TECLE (autores cegados para fines de revisión, 2009). La prueba consistió en 64 ítems con una fiabilidad prueba-reprueba de $r=.88$ basada en normas locales derivadas de una muestra de estudiantes de escuelas primarias de Montevideo (Cuadro, Costa, Trías y Ponce de León, (2009). Esta prueba sólo se administró a los participantes de segundo año, porque no es una 
técnica apropiada para evaluar alumnos de primer año que recién están empezando a aprender a leer.

Comprensión lectora. Se utilizó la subprueba de comprensión lectora de la Batería III Woodcock-Muñoz (Muñoz-Sandoval et al., 2005a). La consistencia interna es $r=.91$ (Schrank et al., 2005).

Programa de Intervención en Alfabetización Multicomponencial (PIAM). El programa de intervención fue diseñado por la primera y segunda autora de este informe, luego de realizar una revisión sistemática de la literatura sobre las intervenciones basadas en la evidencia de alfabetización (Balbi, von Hagen, Cuadrado y Ruiz, 2018). La duración totalizó 15 horas de intervención distribuidas en 20 sesiones de 45 minutos cada una. De este modo durante diez semanas, se desarrollaron dos sesiones de intervención por semana, con grupos de cuatro niños en una habitación tranquila fuera del aula. Diez estudiantes universitarios avanzados de psicología y psicopedagogía fueron seleccionados como tutores para implementar la intervención. Todos habían aprobado tres cursos universitarios enfocados en la evaluación e intervención en alfabetización, cuyos docentes fueron el primer y cuarto autor de este informe. En forma adicional, participaron de una capacitación sobre prácticas de alfabetización basadas en la evidencia diseñada específicamente para este estudio por el primer autor de este artículo. Cada tutor también recibió un manual escrito con los objetivos, la estructura general de la intervención, el desarrollo de cada sesión de entrenamiento, las técnicas a utilizar y los tiempos de intervención del programa.

Diseño de PIAM. Optamos por un diseño multi-componencial centrado en los siguientes cinco componentes principales: conciencia fonológica, instrucción fónica, fluidez, vocabulario y comprensión (National Reading Panel, 2000). Estos cinco componentes fueron entrenados sistemáticamente a través de la implementación de técnicas basadas en la evidencia. Detalles sobre la estructura del programa se presentan en la Tabla 1 y se describen en las siguientes secciones. Elegimos la lectura de cuentos como eje del programa de intervención. Para ello, el primer y segundo autor de este trabajo realizaron una cuidadosa selección de diez cuentos con historias atractivas, motivadoras y con temáticas interesantes para la edad de nuestros participantes. Los cuentos fueron leídos por los tutores y presentados a los participantes en su formato auténtico. 
Eficacia de una intervención en alfabetización basada en la evidencia para niños hispanoparlantes con riesgo lector de niveles socioeconómicos vulnerables

Tabla 1. Diseño del programa de intervención PIAM con dos sesiones modélicas

\begin{tabular}{|c|c|c|c|}
\hline Componentes & Sesión A & Sesión B & Tiempo \\
\hline \multirow{2}{*}{ Comprensión Oral } & Escuchar cuentos & Escalar la montaña & \multirow{2}{*}{8 minutos } \\
\hline & Dialogar con el texto & Preguntas cristalinas y detectivescas & \\
\hline Vocabulario & Palabras nuevas & Definición y uso de nuevas palabras & 8 minutos \\
\hline Conciencia fonológica & Rimas & $\begin{array}{l}\text { Omisión, sustitución, y la inversión de } \\
\text { los fonemas }\end{array}$ & 8 minutos \\
\hline Principio Alfabético & \multicolumn{2}{|c|}{$\begin{array}{l}\text { RCGF: tarjetas con grafemas, sìlabas y palabras, para usar en } \\
\text { tareas de lectura y escritura }\end{array}$} & 8 minutos \\
\hline Fluidez Lectora & $\begin{array}{l}\text { Lectura guiada } \\
\text { Lectura repetida }\end{array}$ & Teatro lector & 8 minutos \\
\hline Autorregulación & Metáfora de un Castill & & 5 minutos \\
\hline
\end{tabular}

Nota. RCGF. Relaciones de Correspondencia entre Grafema y Fonema.

Cada semana el programa comenzaba con la presentación de un nuevo libro de cuentos que motivaba un clima de disfrute hacia la historia. A continuación, seguía la técnica "Dialogar con el texto"' (Hansen y Pearson, 1983) para crear una interacción comunicativa en la que se animaba a los niños a expresar su opinión, activar ideas previas relacionadas con el texto y establecer inferencias. Para la conciencia fonológica e instrucción fónica, utilizamos la técnica de instrucción explícita descrita por Sánchez, Rueda y Orrantia (1989). El tutor verbalizaba cada paso del proceso y modelaba ante los niños. Inmediatamente después, invitaba a cada niño a intentarlo por su cuenta ofreciendo retroalimentación explícita en cada paso del proceso. Los protagonistas de los libros de cuentos aparecieron en cada tarea con el fin de mantener la motivación de los niños. Por ejemplo, un conejo saltaba de un fonema de una palabra a la siguiente o una luz que fallaba y no encendía era usada para omitir fonemas.

Para entrenar la fluidez, utilizamos las técnicas de lectura guiada y lectura repetida (National Reading Panel, 2000). Transcribimos las escenas más emocionantes de los libros simplificando el código, pero sin afectar el sentido, en un cuadernillo de actividades con el que cada niño contaba. Luego pedimos a los niños que leyeran varias veces cada fragmento. Leían a coro, leían representando distintos personajes y cambiaban de rol cada vez y también leían cargando con emociones y prosodias distintas. Para estimular las habilidades de vocabulario seguimos las ideas sugeridas por Beck, McKeown y Kucan (2013). Por ejemplo, seleccionamos 
palabras clave de los cuentos y propusimos tareas de definición, usar las palabras en frases, así como juegos que implicaban buscar sinónimos y antónimos.

Con respecto a la comprensión, el recurso principal que se utilizó fue la metáfora de escalar una montaña (Clarke, Truelove, Hulme y Snowling, 2014). La base de la montaña representaba el inicio del cuento, a medida que escalaban la montaña los niños debían relatar el conflicto de la historia y finalmente bajar la montaña narrando el final o desenlace. También se utilizó la técnica de "preguntas cristalinas y detectivescas" (Jiménez-Fernández, Serrano y Defior, 2014). El tutor planteaba una pregunta que estaba preestablecida en el manual de intervención y los niños tenían que identificar si la información para responderla estaba "clara como el agua"o debían emprender "una búsqueda detectivesca" para resolverla. Por último, cada sesión terminó con una actividad de autorregulación con el objetivo de reflexionar sobre los avances y dificultades que los niños experimentaban a lo largo del proceso de aprendizaje. Utilizamos la técnica de Emmer et al. (2007) empleando la imagen de un castillo, en el que los niños a coloreaban un bloque para cada nueva meta que habían alcanzado.

\section{Procedimiento}

El primer autor de este informe fue contactado por dos fundaciones interesadas en la mejora de las habilidades de alfabetización en estudiantes de escuela primaria de instituciones educativas localizadas en zonas de NSE bajo. En acuerdo con los requerimientos de la Comisión de Ética de la Universidad Católica del Uruguay, recolectamos consentimientos informados por parte de los padres de los participantes de este estudio. La Figura 1 brinda detalles sobre el procedimiento de este estudio. 


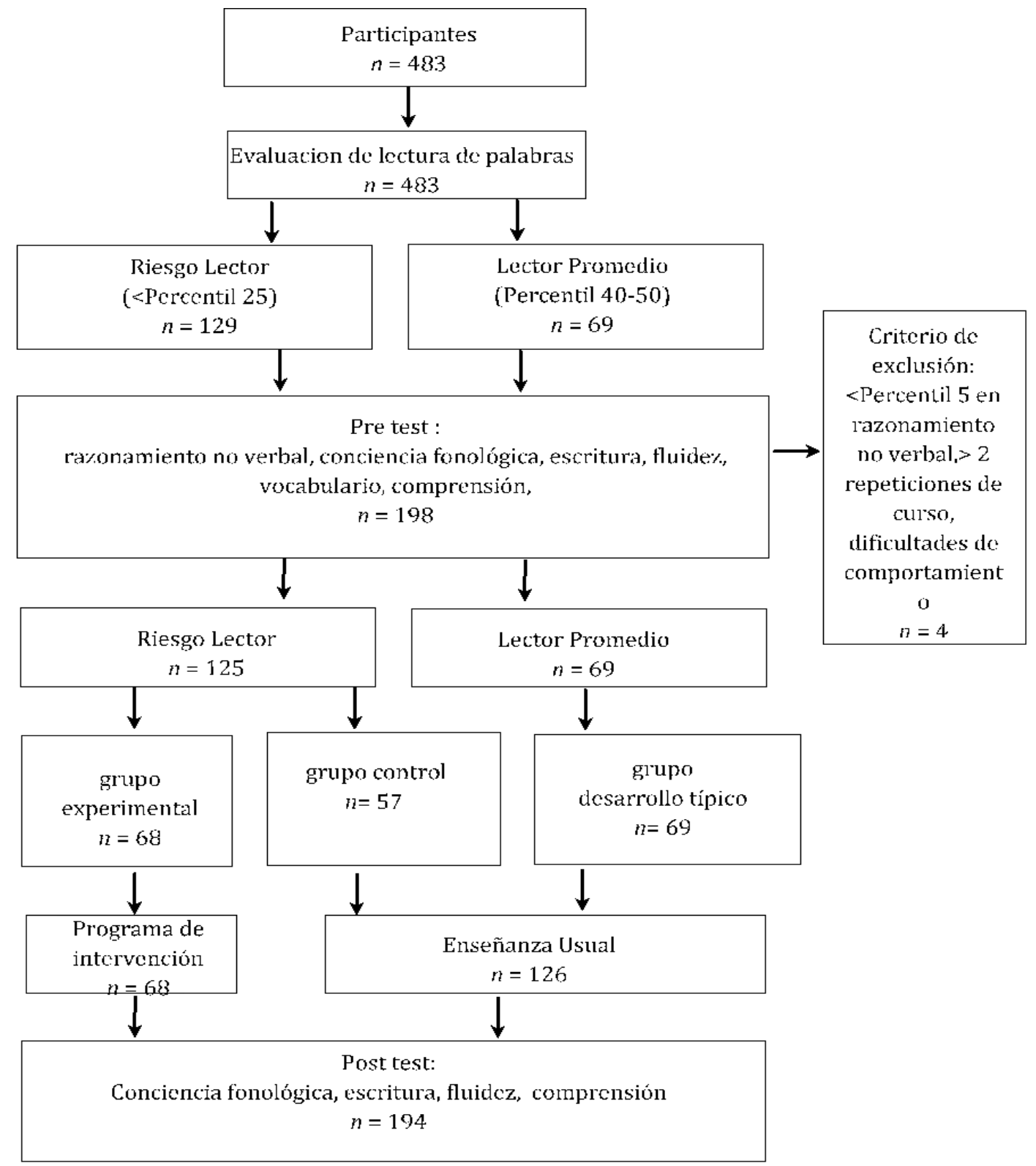

Figura 1. Diagrama de flujo del procedimiento de evaluación e intervención

En primer lugar, evaluamos el nivel lector de la totalidad de alumnos de primer y segundo año de escuela primaria para identificar a los alumnos con riesgo lector y desarrollo lector típico. A continuación, los niños con riesgo lector fueron asignados al azar al $(n=68)$ al grupo experimental que participó de nuestro programa de intervención o al grupo control ( $n=$ 57). Los niños con desarrollo lector típico fueron asignados a un tercer grupo $(n=69)$.

Resultados de análisis de varianza (ANOVA) confirmaron que no había diferencias significativas entre los tres grupos con respecto a la edad, habilidades de razonamiento no 
verbal y comprensión oral. Como se esperaba en base a los criterios de selección, el grupo experimental y control mostraron puntajes significativamente más bajos que el grupo de desarrollo lector típico con respecto a sus habilidades de lectura, $F(2,194)=37.65, p<.001$. El mismo patrón se observó con respecto a las medidas de conciencia fonológica, escritura, fluidez lectora y comprensión lectora. Estos resultados se muestran en la Tabla 2.

Tabla 2. Medidas pre-test y de control

\begin{tabular}{|c|c|c|c|c|c|c|}
\hline Medida & $\begin{array}{c}\mathrm{GE} \\
(n=68)\end{array}$ & $\begin{array}{c}\text { GC } \\
(n=57)\end{array}$ & $\begin{array}{c}\text { GDT } \\
(n=69) \\
\end{array}$ & $F$ & $p$ & $\begin{array}{c}\text { Post-hoc } \\
\text { Bonferroni }\end{array}$ \\
\hline Edad & $6.98(0.06)$ & $7.11(0.09)$ & $6.98(0.06)$ & 0.05 & 0.62 & - \\
\hline $\begin{array}{l}\text { Razonamiento no } \\
\text { verbal }\end{array}$ & $18.73(4.47)$ & $18.12(4.53)$ & $19.77(4.93)$ & 2.06 & 0.62 & - \\
\hline Comprensión Oral & $14.45(3.65)$ & $14.81(4.03)$ & $15.61(4.27)$ & 1.53 & 0.94 & - \\
\hline $\begin{array}{l}\text { Habilidades de } \\
\text { lectura }\end{array}$ & $12.11(7.18)$ & $12.21(7.17)$ & $24.29(12.40)$ & $\begin{array}{c}37.6 \\
5\end{array}$ & $<.01 * *$ & $\begin{array}{l}\mathrm{GE}<\mathrm{GDT} \\
\mathrm{GC}<\mathrm{GDT}\end{array}$ \\
\hline $\begin{array}{l}\text { Conciencia } \\
\text { fonológica }\end{array}$ & $7.11(4.95)$ & $7.46(5.3)$ & $9.10(7.26)$ & 6.42 & $<.01 * *$ & $\begin{array}{l}\mathrm{GE}<\mathrm{GDT} \\
\mathrm{GC}<\mathrm{GDT}\end{array}$ \\
\hline Escritura & $13.56(7.50)$ & $15.28(8.14)$ & $18.83(7.86)$ & 8.62 & $<.001 * * *$ & $\begin{array}{l}\text { GE }<\text { GDT } \\
\text { GC }<\text { GDT }\end{array}$ \\
\hline Fluidez lectora & $4.10(1.98)$ & $4.31(2.52)$ & $11.11(4.21)$ & $\begin{array}{c}47.1 \\
8\end{array}$ & $<.001 * * *$ & $\begin{array}{l}\text { GE }<\text { GDT } \\
\text { GC }<\text { GDT }\end{array}$ \\
\hline $\begin{array}{l}\text { Comprensión } \\
\text { Lectora }\end{array}$ & $6.25(2.68)$ & $7.77(3.75)$ & $12.53(5.4)$ & $\begin{array}{c}38.6 \\
3\end{array}$ & $<.001 * * *$ & $\begin{array}{l}\mathrm{GE}<\mathrm{GDT} \\
\mathrm{GC}<\mathrm{GDT}\end{array}$ \\
\hline
\end{tabular}

Nota GE = grupo experimental; GC = grupo control; GDT = grupo de desarrollo típico; todos los valores son reportados en puntuaciones directas con la excepción de la variable edad, que se informa en años; Desviaciones estándar se reportan en paréntesis. $* p<.05$, dos colas; ** $p<.01$, dos colas; *** $p<.001$, dos colas.

Sólo el grupo experimental con riesgo lector recibió una intervención de alfabetización suplementaria fuera del aula, mientras el grupo control y el grupo de desarrollo lector típico permanecieron en sus clases regulares y recibieron “enseñanza usual” por parte de sus maestros. Una vez completada la intervención, tomamos medidas post-test en conciencia fonológica, escritura de palabras, fluidez lectora y comprensión lectora para los tres grupos de participantes. Ninguno de los participantes abandonó el estudio y la asistencia fue superior al $95 \%$ en todas las instancias de evaluación e intervención. 


\section{Resultados}

En primer lugar, se realizó un análisis de varianza (ANOVA) de medidas repetidas comparando los puntajes pre-post en las pruebas de conciencia fonológica, escritura, fluidez lectora y comprensión lectora con el fin de evaluar los avances logrados por cada grupo de participantes. Los resultados se detallan en la Tabla 3 y revelaron una mejora pre-post-test significativa con efectos de tamaño medio (Cohen, 1988) para los tres grupos de participantes en las cuatro variables dependientes analizadas.

Tabla 3. Medidas pre y post-test

\begin{tabular}{llllll}
\hline Grupo experimental & Pre-test & Post-test & \multicolumn{2}{l}{ ANOVA } & \\
Medida & $M(S D)$ & $M(S D)$ & $F \quad p$ & $\eta 2$ \\
\hline CF & $7.11(4.95)$ & $15.24(6.59)$ & $205.81<.001 * * *$ & 0.71 \\
Escritura & $13.56(7.47)$ & $21.90(6.13)$ & $141.50<.001 * * *$ & 0.68 \\
Fluidez lectora & $4.10(1.98)$ & $8.79(3.63)$ & $55.42<.001 * * *$ & 0.66 \\
Comprensión lectora & $6.25(2.66)$ & $14.16(6.4)$ & $124.60<.001 * * *$ & 0.68 \\
\hline Grupo control & Pre-test & Post-test & ANOVA & \\
Medida & $M(S D)$ & $M(S D)$ & $F \quad p$ & $\eta 2$ \\
\hline CF & $7.46(5.27)$ & $12.35(6.19)$ & $48.57<.001 * * *$ & 0.46 \\
Escritura & $15.28(8.14)$ & $21.58(6.62)$ & $66.42<.001 * * *$ & 0.54 \\
Fluidez lectoraa & $4.31(2.52)$ & $7.81(3.33)$ & $31.53<.001 * * *$ & 0.56 \\
Comprensión lectora & $7.77(3.75)$ & $13.09(6.11)$ & $105.82<.001 * * *$ & 0.76 \\
\hline Grupo Normotípico & Pre-test & Post-test & ANOVA & \\
$M e d i d a$ & $M(S D)$ & $M(S D)$ & $F$ & $p$ & $\eta 2$ \\
\hline CF & $9.10(7.62)$ & $17.74(7.93)$ & $205.81<.001 * * *$ & 0.76 \\
Escritura & $18.83(7.86)$ & $26.46(5.62)$ & $217,39<.001 * * *$ & 0.76 \\
Fluidez lectora & $11.11(4.22)$ & $16.11(4.69)$ & $65.54<.001 * * *$ & 0.71 \\
Comprensión lectora & $12.53(5.49)$ & $20.14(5.3)$ & $208.81<.001 * * *$ & 0.76 \\
\hline
\end{tabular}

Nota $. \mathrm{CF}=$ Conciencia fonológica; ${ }^{a}$ Los resultados para la medida fluidez se basaron solamente en alumnos de $2^{\circ}$ año escolar $(n=83) ;{ }^{*} p<.05$, dos colas; $* * p<.01$, dos colas; **** $p<.001$, dos colas.

El segundo análisis tuvo por finalidad explorar las mejoras específicas del grupo experimental con riesgo lector, en comparación con el grupo control y el grupo de desarrollo lector típico. Por lo tanto, realizamos análisis de co-varianza (ANCOVA) para las variables dependientes conciencia fonológica, escritura, fluidez lectora y comprensión lectora controlando por los puntajes pre-test en estas mismas medidas. En conciencia fonológica, los resultados reflejaron una diferencia significativa entre los puntajes post-test alcanzados por los tres grupos de participantes, $F(2,181)=10.3, p<.001, \eta^{2}=.101$. Análisis post-hoc de 
Bonferroni confirmaron que el grupo experimental de riesgo lector $(M=15.24 ; S D=6.59)$ logró puntajes post-test significativamente mejores que el grupo control $(M=12.35 ; S D=6.19)$, $p<.001$. Además, solamente el grupo control, pero no el grupo experimental, continuó mostrando un rendimiento significativamente por debajo del grupo de desarrollo lector típico en esta medida, $p<.001$.

Un análisis más detallado de las diferentes sub-habilidades de conciencia fonológica que evaluamos (rima, omisión, sustitución e inversión de fonemas), mostró diferencias significativas entre los grupos de participantes en estas cuatro variables dependientes. Análisis post-hoc de Bonferroni revelaron que el grupo experimental alcanzó avances significativamente superiores al grupo control en la sub-habilidad identificación de rimas, pero no en la omisión, sustitución e inversión de fonemas. Tanto el grupo experimental, como el grupo control progresaron significativamente menos en las sub-habilidades de omisión, sustitución e inversión de fonemas que el grupo de desarrollo lector típico. Estos resultados se detallan en la Tabla 4.

Tabla 4. Medidas pre-post-test para sub-habilidades de conciencia fonológica

\begin{tabular}{|c|c|c|c|c|c|c|c|c|c|}
\hline \multirow{2}{*}{ Variable } & \multicolumn{3}{|c|}{ Pre-test } & \multicolumn{3}{|c|}{ Post-test } & \multicolumn{3}{|c|}{ ANCOVA } \\
\hline & GE & GC & GDT & GE & GC & GDT & $F$ & $p$ & Bonferroni \\
\hline Rima & $\begin{array}{c}2.96 \\
(1.67)\end{array}$ & $\begin{array}{c}3.12 \\
(1.78)\end{array}$ & $\begin{array}{l}4.26 \\
(2.24)\end{array}$ & $\begin{array}{c}6.43 \\
(2.49)\end{array}$ & $\begin{array}{l}4.39 \\
(2.07)\end{array}$ & $\begin{array}{c}5.87 \\
(2.34)\end{array}$ & 13.42 & $<.001 * * *$ & $\begin{array}{c}\mathrm{GC}<\mathrm{GE} \\
\mathrm{GC}< \\
\mathrm{GDT}\end{array}$ \\
\hline $\begin{array}{l}\text { Omisión de } \\
\text { fonemas }\end{array}$ & $\begin{array}{c}1.56 \\
(1.50)\end{array}$ & $\begin{array}{c}1.63 \\
(1.65)\end{array}$ & $\begin{array}{c}1.63 \\
(1.65)\end{array}$ & $\begin{array}{c}2.81 \\
(1.85)\end{array}$ & $\begin{array}{c}2.81 \\
(1.85)\end{array}$ & $\begin{array}{c}4.29 \\
(1.95)\end{array}$ & 9.67 & $<.001 * * *$ & $\begin{array}{l}\text { GE < } \\
\text { GDT } \\
\text { GC < } \\
\text { GDT }\end{array}$ \\
\hline $\begin{array}{l}\text { Sustitución } \\
\text { de fonemas }\end{array}$ & $\begin{array}{c}0.91 \\
(0.94)\end{array}$ & $\begin{array}{c}0.98 \\
(0.99)\end{array}$ & $\begin{array}{c}1.70 \\
(1.67)\end{array}$ & $\begin{array}{c}2.34 \\
(1.80)\end{array}$ & $\begin{array}{l}1.95 \\
(1.43)\end{array}$ & $\begin{array}{c}3.45 \\
(2.67)\end{array}$ & 6.71 & $<.001 * * *$ & $\begin{array}{l}\text { GE < } \\
\text { GDT } \\
\text { GC < } \\
\text { GDT }\end{array}$ \\
\hline $\begin{array}{l}\text { Inversión de } \\
\text { fonemas }\end{array}$ & $\begin{array}{c}1.44 \\
(1.60)\end{array}$ & $\begin{array}{c}1.72 \\
(1.73)\end{array}$ & $\begin{array}{c}2.24 \\
(2.41)\end{array}$ & $\begin{array}{l}3.43 \\
(2.59)\end{array}$ & $\begin{array}{c}3.32 \\
(2.56)\end{array}$ & $\begin{array}{c}5.19 \\
(2.42)\end{array}$ & 6.48 & $<.001 * * *$ & $\begin{array}{l}\text { GE < } \\
\text { GDT } \\
\text { GC < } \\
\text { GDT }\end{array}$ \\
\hline
\end{tabular}

Nota GE = grupo experimental; GC = grupo control; GDT = grupo de desarrollo lector típico; $* p<.05$, dos colas; $* * p<.01$, dos colas; $* * * p<.001$, dos colas.

En cuanto a la variable dependiente escritura, también encontramos diferencias significativas entre los grupos de participantes, $F(2,193)=10.85, p<.001, \eta^{2}=.107$. Más específicamente, el grupo experimental $(M=21.90 ; S D=6.13)$ y el grupo control $(M=21.58$; 
$S D=6.68)$ continuaron rindiendo significativamente por debajo del grupo de desarrollo lector típico $(M=26.97 ; S D=4.93)$, según análisis post-hoc de Bonferroni. No encontramos diferencias entre el rendimiento del grupo experimental y control en esta medida.

Para el caso de la variable dependiente fluidez lectora, solamente contamos con datos de los niños de $2^{\circ}$ año escolar $(n=83)$. Nuevamente, observamos diferencias significativas entre los tres grupos de participantes en esta medida, $F(2,79)=6.36, p<.01, \eta^{2}=.139$. El grupo de desarrollo lector típico $(M=16.11 ; S D=4.69)$ mostró puntajes significativamente más altos, que el grupo control $(M=7.81 ; S D=3.33)$ por un lado, y que el grupo experimental $(M=8.79$; $S D=3.63$ ) por otro lado, según revelaron análisis post-hoc de Bonferroni, $p=.032$ y $p=.002$, respectivamente.

Finalmente, observamos diferencias significativas entre los tres grupos de participantes en la variable dependiente de comprensión lectora, $F(2,182)=3.98, p<.05, \eta^{2}=.048$. Los resultados de análisis post-hoc de Bonferroni indicaron que el grupo control $(M=13.09 ; S D=$ 6.11) continuó rindiendo significativamente por debajo del grupo de desarrollo típico $(M=$ 20.14; $S D=5.3), p<.05$. Sin embargo, no detectamos diferencias significativas entre el rendimiento del grupo experimental $(M=13.68 ; S D=6.4)$ y el grupo de control, por un lado, y el grupo de desarrollo lector típico, por otro lado.

\section{Discusión y Conclusiones}

El objetivo del presente estudio fue investigar en qué medida una intervención de alfabetización basada en la evidencia derivada de la investigación con poblaciones de habla inglesa, podía ser pertinente para mejorar las habilidades de alfabetización en niños con riesgo lector de NSE bajo en países de habla hispana. Aunque nuestros participantes mostraron mejoras significativas en el post-test, el progreso de los niños del grupo experimental sólo pudimos atribuirlo a nuestra intervención en la sub-habilidad de identificación de rimas y parcialmente en la habilidad de comprensión lectora. Nuestros resultados son consistentes con la evidencia proporcionada por estudios similares realizados en otros países de habla hispana en América Latina (Pallante y Kim, 2013; Strasser et al, 2016.; Yoshikawa et al., 2015) y contrastan con los resultados de investigaciones con participantes de habla hispana en EE.UU. (Escamilla et al., 1998; Mathes et al., 2007). Se discuten cuatro posibles explicaciones. 
En primer lugar, las diferencias entre los sistemas de escritura del español e inglés pueden imponer diferentes demandas en las habilidades que deben ser enseñadas a los lectores en riesgo. Por ejemplo, todavía no está claro, si la conciencia fonológica es tan eficaz para mejorar las habilidades de alfabetización en ortografías poco profundas como el español como en inglés. Ehri et al. (2001) informa sólo pequeños tamaños del efecto para lenguas distintas al inglés. Furnes y Samuelson (2010) encontraron que la conciencia fonológica podía ser un predictor significativo de la capacidad de lectura sólo hasta el primer año de la escuela primaria en participantes suecos y noruegos. También Tafa y Manolitsis (2008) llegaron a la conclusión de que la conciencia fonológica no contribuyó en la distinción entre lectores en riesgo y lectores promedio en lengua griega. Por tanto, la intervención en conciencia fonológica podría requerir más intensidad en cuanto duración y énfasis en habilidades críticas de conciencia fonémica para mejorar habilidades de alfabetización en niños hispanoparlantes. Esto podría explicar por qué la mejora en conciencia fonológica que observamos en nuestros participantes no se tradujo en mejoras específicas en otras medidas de alfabetización.

A su vez, las distintas lenguas podrían imponer diferentes demandas de las subhabilidades de conciencia fonológica que deben ser enseñadas a los lectores en riesgo. En español se ha demostrado que unidades fonológicas más pequeñas, como las sílabas y fonemas, en lugar de unidades más grandes, como la rima, podrían jugar un papel más importante (Goodwin et al., 2015). La instrucción de habilidades de rima en español, por lo tanto, podría ser irrelevante para mejorar las habilidades de alfabetización de participantes hispanoparlantes. Al igual que nosotros, Guardia (2014) no encontró asociaciones significativas entre la capacidad de la rima y medidas de lectura de precisión, velocidad y comprensión en estudiantes de primer año de la escuela primaria en Chile. Considerando que las habilidades de rima evolutivamente son anteriores al desarrollo de la conciencia fonémica, también es posible interpretar nuestros resultados como evidencia del inicio de un cambio cognitivo, que aún no alcanza a mostrarse en una mejora significativa en la manipulación de fonemas. Quizás el tiempo dedicado a entrenar habilidades de conciencia fonémica en nuestra intervención no haya sido suficiente para generar este impacto.

En segundo lugar, nuestra decisión de diseñar un plan de alfabetización multicomponencial asignando un tiempo de instrucción similar a cada componente podría haber limitado el impacto de nuestra intervención. En español hay estudios que señalan que la fluidez lectora requiere entrenamiento específico y sostenido (Castejón, Gonzalez-Pumariega, Cuetos, 
2015). También Gersten et al. (2008) sugieren que es necesario centrarse en sólo unas pocas habilidades centrales con el fin de obtener medidas de impacto significativo, argumentando que podría ser especialmente difícil para niños con riesgo lector, dar prioridad a las habilidades más relevantes si se presentan demasiados objetivos de aprendizaje. El número de las habilidades incluidas en una intervención de alfabetización tiene importantes consecuencias sobre el tiempo asignado a cada habilidad objetivo. Por ejemplo, nosotros dedicamos 8 minutos por sesión para enseñar cada una de las cinco habilidades objetivo, totalizando 2 horas y 40 minutos a lo largo de toda la intervención para cada componente. Esto podría no haber sido suficiente para impactar en mejoras específicas. Intervenciones de alfabetización en español que mostraron un impacto positivo realizaron sesiones de entrenamiento más intensas. Mathes et al. (2007) realizó sesiones diarias de 50 minutos durante 25 semanas. En forma similar, el carácter suplementario de nuestra intervención en comparación con intervenciones que sustituyen la enseñanza usual en el aula podría ser otra razón para explicar el impacto limitado que observamos. Wanzek y Vaughn (2007) apuntan a la necesidad de considerar las limitaciones prácticas del contexto educativo cuando se debate sobre la duración de los programas de intervención. Este aspecto es especialmente importante en este estudio, que fue realizado en contextos educativos auténticos con expectativas de impacto educativo y social, condición que impone necesidades de administración eficiente de recursos limitados. Por lo tanto pensamos que podría ser preferible realizar intervenciones de alfabetización más intensas con un menor número de niños en proyectos futuros.

En tercer lugar, el bajo nivel socioeconómico de los participantes involucrados en este estudio podría haber limitado el impacto que hemos observado como resultado de nuestra intervención. En coherencia con Strasser et al. (2016) creemos que los participantes de nuestro estudio podrían haber mostrado déficits de desarrollo y lingüísticos más amplios y profundos que los niños de NSE bajo en estudios similares con participantes angloparlantes. Como Al Otaiba y Fuchs (2002) indican, es más probable que estudiantes de contextos minoritarios presentan dificultades asociadas y trastornos comórbidos.

En cuarto lugar, el impacto limitado de nuestra intervención podría explicarse por una difusión de la información de las características de la intervención experimental a los docentes de la enseñanza usual en el aula (Gunderson y Svartdal, 2010). Este concepto, descrito originalmente como difusión de tratamiento, se refiere a la filtración de información sobre la intervención entre los profesores regulares. Como mencionamos, nuestra investigación estaba 
inserta en un contexto educativo auténtico con ambiciones amplias de mejora de las habilidades de alfabetización para las seis escuelas participantes, subvencionado por dos fundaciones. En este contexto, se alentó a los maestros de las clases regulares a mejorar sus estrategias de instrucción de alfabetización quienes recibieron una jornada de capacitación general sobre alfabetización por los dos primeros autores de este artículo. Esto podría haber influido en la enseñanza usual de los docentes, que tal vez incorporaron prácticas de alfabetización basadas en la evidencia, que podrían tener semejanza con nuestro programa de intervención. En una situación similar, Duff, Hulme, Grainger, Hardwick, Miles y Snowling (2014) informan de la contaminación de su grupo control debido a la influencia ya no solo de los profesores, sino también de los padres que fueron informados sobre los objetivos de la instrucción.

En resumen, el presente estudio se centró en examinar la eficacia de una intervención en alfabetización basada en la evidencia para niños hispanoparlantes con riesgo lector de niveles socioeconómicos vulnerables. Una de las preguntas centrales que motivó este estudio fue indagar si una intervención diseñada a partir de principios basados en la evidencia proveniente de estudios de investigación con participantes angloparlantes también es adecuada para mejorar las habilidades de alfabetización de niños hispanoparlantes. Aunque los participantes de nuestra intervención mejoraron significativamente sus habilidades de conciencia fonológica, escritura, fluidez y comprensión, estos resultados solamente pudieron ser adjudicados en forma específica a nuestra intervención con respecto a habilidades de rima y parcialmente a habilidades de comprensión. En línea con investigaciones pasadas, nuestros hallazgos señalan la necesidad de tomar en cuenta los contrastes entre los sistemas de escritura del español y el inglés, así como el perfil cognitivo de niños de NSE vulnerables en Lationamerica para explicar resultados inconsistentes en el impacto alcanzado por intervenciones en alfabetización realizados en países anglo- e hispanoparlantes.

\section{Referencias}

Al Otaiba, S., y Fuchs, D. (2002). Characteristics of children who are unresponsive to early literacy intervention. A review of the literature. Remedial and Special Education, 23(5), 300-316. doi: 10.1177/07419325020230050501

Balbi, A., von Hagen, A., Cuadro, A. y Ruiz, C. (2018). Revisión sistemática sobre intervenciones en alfabetización temprana: implicancias para intervenir en español. Revista Latinoamericana de Psicología, 50 (1), 31-48. 
Beck, I. L., McKeown, M. G., y Kucan, L. (2013). Bringing words to life: Robust vocabulary instruction. New York: Guilford Press.

Caravolas, M., Lervag, A., Mousikou, P., Efrim, C., Litavsky, M., Onochie-Quintanilla, E. et al. (2012). Common Patterns of Prediction of Literacy Development in Different Alphabetic Orthographies. Psychological Science 23(6), 678-686. doi: $10.1177 / 0956797611434536$

Castejón, L., González-Pumariega, S. y Cuetos, F. (2015) Development of word reading fluency along primary education: a six-year follow-up / El desarrollo de la fluidez en la lectura de palabras en educación primaria: un seguimiento longitudinal de seis años, Infancia y Aprendizaje, 38:4, 842-871,DOI: 10.1080/02103702.2015.1076266

Clarke, P.J., Truelove, E., Hulme, C. y Snowling, M.J. (2014). Developing Reading Comprehension. Chichester: Wiley-Blackwell.

Cohen, J. (1988). Statistical power analysis for the behavioural sciences (2nd ed.) New York: Academic Press.

Defior, S., Jiménez-Fernández, G., Calet, N., y Serrano, F. (2015). Learning to read and write in Spanish: phonology in addition to which other processes?/Aprendiendo a leer y escribir en español: además de la fonología, ¿ qué otros procesos? Estudios de Psicología, 36(3), 571-591. doi: 10.1080/02109395.2015.1078552

Duff, F. J., Hulme, C., Grainger, K., Hardwick, S. J., Miles, J. N., y Snowling, M. J. (2014). Reading and language intervention for children at risk of dyslexia: a randomised controlled trial. Journal of Child Psychology and Psychiatry, 55(11), 1234-1243. doi: $10.1111 / \mathrm{jcpp} .12257$.

Ehri, L. C., Nunes, S. R., Willows, D. M., Schuster, B. V., Yaghoub - Zadeh, Z., y Shanahan, T. (2001). Phonemic awareness instruction helps children learn to read: Evidence from the National Reading Panel's meta-analysis. Reading research quarterly, 36(3), 250287. doi: 10.1598/RRQ.36.3.2.

Emmer, A., Hofmann, B., y Matthes, G. (2007). Elementares Training bei Kindern mit Lernschwierigkeiten: Test-und Trainingsverfahren. [Elementary training for children with learning difficulties: test and training procedure]. Weinheim: Beltz.

Escamilla, K., Loera, M., Ruiz, O., y Rodríguez, Y. (1998). An examination of sustaining effects in Descubriendo la Lectura programs. Literacy, Teaching and Learning, 3(2), 59. 
Furnes, B., y Samuelsson, S. (2010). Predicting reading and spelling difficulties in transparent and opaque orthographies: A comparison between Scandinavian and US/Australian children. Dyslexia, 16(2), 119-142. doi: 10.1002/dys.401

Gersten, R., Compton, D., Connor, C. M., Dimino, J., Santoro, L., Linan-Thompson, S., y Tilly, W. D. (2008). Assisting students struggling with reading: Response to Intervention and multi-tier intervention for reading in the primary grades. A practice guide (NCEE 20094045). Washington, DC: National Center for Education Evaluation and Regional Assistance, Institute of Education Sciences, US Department of Education.

Goodwin, A. P., August, D., y Calderon, M. (2015). Reading in Multiple Orthographies: Differences and Similarities in Reading in Spanish and English for English Learners. Language Learning, 65(3), 596-630. doi: 10.1111/lang.12127.

Guardia, P. (2014). Is consonant rhyme important when learning to read in Spanish?/¿Es importante la rima consonante para aprender a leer en el español? Estudios de Psicología, 35(3), 567-583. doi: 10.1080/02109395.2014.965459

Gundersen, K., y Svartdal, F. (2010). Diffusion of treatment interventions: exploration of 'secondary' treatment diffusion. Psychology, Crime y Law, 16(3), 233-249. doi: $10.1080 / 10683160802612924$

Hansen, J. y Pearson , P.D. (1983). An instructional study: Improving the inferential comprehension of fourth grade good and poor readers. Journal of Educational Psychology, 75, 821-829. doi: 10.1037/0022-0663.75.6.821

INE (2012) Indice de Nivel Socioeconómocio. [Index of Socio-economic Status]. Retrieved from http://www.gruporadar.com.uy/01/wp-content/uploads/2012/04/Informe-NuevoINSE-2011.pdf

Jara Quezada, N. y Troncoso San Martín, J. (2014). Validación Test de Matrices Progresivas de Raven Escala coloreada, en escolares de la ciudad de Chillán. [Validation of the Test of Progressive Matrices - Coloured Scale in primary school students of Chillán]. Bachelor Thesis of Psychology. Universidad del Bío-Bío. Retrieved from http://repobib.ubiobio.cl/jspui/handle/123456789/204.

Jiménez-Fernández, G.; Serrano, F. y Defior, S. (2014). Elaboración de material audiovisual para la mejora de la docencia práctica de asignaturas relacionadas con el lenguaje escrito (PID 11-01) [Design of audiovisual materials to improve teaching of subjects related to written language (PID 11-01)]. In L. Jiménez del Barco Jaldo y M. C. García Garnica (coords.), Innovación docente y buenas prácticas en la Universidad de Granada, vol 3 
[Teaching innovation and good practices at the University of Granada] (pp. 283-295). Granada: Editorial Universidad de Granada.

Kohnen, S., Colenbrander, D., Krajenbrink, T., y Nickels, L. (2015). Assessment of lexical and non-lexical spelling in students in Grades 1-7. Australian Journal of Learning Difficulties, 20(1), 15-38.

Mathes, P. G., Pollard-Durodola, S. D., Cárdenas-Hagan, E., Linan-Thompson, S., y Vaughn, S. (2007). Teaching struggling readers who are native Spanish speakers: what do we know? Language, Speech, and Hearing Services in Schools, 38(3), 260-271. doi: 10.1044/0161-1461(2007/027)

Moll, K., Ramus, F., Bartling, J., Bruder, J., Kunze, S., Neuhoff, N. et al. (2014). Cognitive mechanisms underlying reading and spelling development in five European orthographies. Learning and Instruction, 29, 65-77. doi: 10.1016/j.learninstruc.2013.09.003

Muñoz-Sandoval. A. F.; Woodcock, R.W.; McGrew, K.S. y Mather, N. (2005a). Batería III Woodcock-Muñoz: Pruebas de habilidades cognitivas. [Woodcock-Muñoz Battery III: Tests of cognitive abilities.]. Spring Lake Drive, Itasca: The Riverside Publishing Company.

National Reading Panel (US), National Institute of Child Health, y Human Development (US). (2000). Report of the national reading panel: Teaching children to read: An evidencebased assessment of the scientific research literature on reading and its implications for reading instruction: Reports of the subgroups. Retrieved from https://www.nichd.nih.gov/publications/pubs/nrp/documents/report.pdf

Pallante, D. H., y Kim, Y. S. (2013). The effect of a multicomponent literacy instruction model on literacy growth for kindergartners and first-grade students in Chile. International Journal of Psychology, 48(5), 747-761. doi: 10.1080/00207594.2012.719628.

Raven, J.C. (1993). Test de Matrices Progresivas. Cuaderno de Matrices, Escala Coloreada. Series A, Ab y B. [Test of Progressive Matrices - Coloured Scale. Series A, Ab and B]. Buenos Aires: Paidós.

Sánchez, E., Rueda, M. I., y Orrantia, J. (1989). Estrategias de intervención para la reeducación de niños con dificultades en el aprendizaje de la lectura y de la escritura. [Intervention strategies for training children with Reading and writing difficulties]. Comunicación, lenguaje y educación, 1(3-4), 101-111.

Schrank, F. A., McGrew, K. S., Ruef, M. L., Alvarado, C. G., Muñoz-Sandoval, A. F., y Woodcock, R. W. (2005). Overview and technical supplement (Batería III Woodcock- 
Muñoz Assessment Service Bulletin No. 1). Rolling Meadows, IL: Riverside Publishing.

Share, D. L. (2008). On the Anglocentricities of current reading research and practice: the perils of overreliance on an" outlier" orthography. Psychological bulletin, 134(4), 584. doi: 10.1037/0033-2909.134.4.584.

Strasser, K., Rolla, A., y Romero-Contreras, S. (2016). School Readiness Research in Latin America: Findings and Challenges. New directions for child and adolescent development, 2016, (152), 31-44. doi: 10.1002/cad.20160.

Tafa, E., y Manolitsis, G. (2008). A longitudinal literacy profile of Greek precocious readers. Reading Research Quarterly, 43(2), 165-185. doi: 10.1598/RRQ.43.2.4

UCU (2012). Database of private primary schools fees in Montevideo, Uruguay. Unpublished. Montevideo: Universidad Católica del Uruguay.

Wanzek, J., y Vaughn, S. (2007). Research-based implications from extensive early reading interventions. School Psychology Review, 36(4), 541.

Yoshikawa, H., Leyva, D., Snow, C. E., Treviño, E., Barata, M., Weiland, C., ... y Arbour, M. C. (2015). Experimental impacts of a teacher professional development program in Chile on preschool classroom quality and child outcomes. Developmental psychology, 51(3), 309. doi: 10.1037/a0038785

Recibido: $26-08-2019$

Aceptado: 18-11-2019 